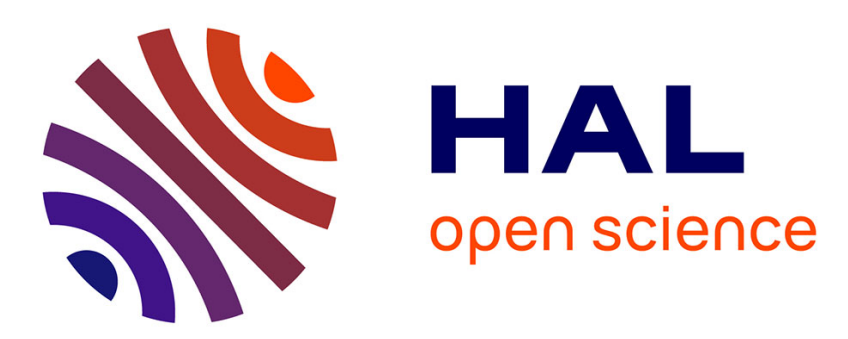

\title{
The fact-theory dialogue in an industrial context: the case of statistical quality control
}

\author{
Denis Bayart
}

\section{To cite this version:}

Denis Bayart. The fact-theory dialogue in an industrial context: the case of statistical quality control.

European Management Review, 2006, 3, pp.87-99. hal-00263050

\section{HAL Id: hal-00263050 \\ https://hal.science/hal-00263050}

Submitted on 11 Mar 2008

HAL is a multi-disciplinary open access archive for the deposit and dissemination of scientific research documents, whether they are published or not. The documents may come from teaching and research institutions in France or abroad, or from public or private research centers.
L'archive ouverte pluridisciplinaire HAL, est destinée au dépôt et à la diffusion de documents scientifiques de niveau recherche, publiés ou non, émanant des établissements d'enseignement et de recherche français ou étrangers, des laboratoires publics ou privés. 
THE FACT-THEORY DIALOGUE IN AN INDUSTRIAL CONTEXT. THE CASE OF STATISTICAL QUALITY CONTROL.

To be published in European Manaqement Review, 2006

\author{
Denis Bayart \\ Centre de recherche en gestion (PREG-CRG) \\ Ecole polytechnique et CNRS \\ Paris, France \\ 33(1) 55558321 \\ 33(1) 55558444 (fax) \\ denis.bayart@polytechnique.edu
}




\begin{abstract}
Statistical control of quality provides a useful example of the way that industry practices a pragmatic-scientific approach for managing production operations. Knowledge, applied to action, has circumscribed theories with means-end constraints, feedback deadlines, and information economies. These have, surprisingly, led to further original and fruitful theoretical questions. Moreover, the need to adapt scientific methods to a little-qualified workforce produced innovative cognitive tools. This paper studies these aspects of the knowledge creation process in an industrial activity and shows that the metaphor of a "dialogue" organized between facts and theory is, in this context, more accurate than the model of hypothesis testing and planned experiment.
\end{abstract}

Keywords:

empirical knowledge creation, epistemology, pragmatism, cognitive tools, cost of information, statistical quality control, Deming cycle. 
"But when do symbols mean the same to a given group of people? The modern logician will likely answer that a symbolic statement means the same to two or more persons when it leads to the same action on the part of each of them." (Shewhart, 1933)

The process of knowledge creation in firms will be studied here under the aspect of the organization of interactions with the empirical world. The sought-after knowledge bears upon this empirical world and is to be used for economic or managerial purposes. Examples can be found in various management domains such as strategy, marketing, human resources, operations management, in those instances where specific investigations have to be led in order to acquire knowledge. At a time where knowledge management is such a wide field of research, it is necessary to get a deeper understanding of the process of knowledge creation in firms by identifying types of knowledge and knowledge-creation processes. To contribute to the specification of the knowledge-creation process, we propose to take into account two kinds of factors : first, epistemological properties of the knowledge which relate to properties of the empirical field of study and to properties of the methodological tools put to use; second, the organizational and managerial context of the production of knowledge, especially the conditions to be fulfilled so that it be valuable to management.

This first factor does not seem to have been widely studied so far in organizational studies. Knowledge creation is a phenomenon often studied by management scholars but usually without taking into account the epistemic dimension of the knowledge, i.e. what kind of knowledge it is, how it is acquired, how it relates to the empirical world it describes. For example, the famous studies by Nonaka \& Takeuchi, (1995) insist on making explicit knowledge but do not emphasize the way tacit knowledge is produced. In most cases, tacit knowledge is supposed to arise spontaneously from practice. The communities of practice approach (Brown \& Duguid, 1991) focuses on practice and on the socialization of knowledge but does not consider the epistemic specificities of knowledge. In that instance, one sees perfectly well how situated 
knowledge is produced between the actors (repairmen) through recollection and storytelling but no specific properties of this knowledge are put forward, which could explain why it is suitable to this kind of working together.

In constrast, investigations led by sociologists of science adopting an ethnographic approach (Knorr Cetina, 1999) show that the communities of scientists are very differently structured according to the discipline they work in, notably on account of the relation to the empirical world. The concept of "epistemic culture" refers to "those amalgams of arrangements and mechanisms-bonded through affinity, necessity, and historical coincidence-which, in a given field, make up how we know what we know" (Knorr Cetina, 1999, 1). This author shows that different sciences enforce quite different attitudes and methods toward empirical phenomena. In this respect, the comparison of particle physics and molecular biology is striking. Ethnographic analyses of the scientific activity do not often study "the empirical machinery used in the creation of knowledge", which is however the most practical demonstration of epistemological and methodological differences between scientific specialities.

Transposing this line of reasoning to the domain of management, we find for example strong differences between the epistemic cultures of marketing departments and of production departments due to very different relations with the objects of study. Questions can be raised concerning the effect of such variance upon knowledge management theories and practice. Considering the knowledge contents seemingly leads to new insights about the organizational life of knowledge. We suggest that knowledge management should be aware, at the same time, of the specificities of the knowledge to manage and of the expectations of management regarding its use, so that a fit could be found between both aspects. Here we show an example where such a fit is designed into the empirical machinery of the knowledge creation process itself.

Our hypotheses are grounded in the case study of Statistical Quality Control (SQC), an illustration of a successful fit between epistemological and managerial aspects of knowledge. The SQC movement started at AT\&T in 1922 at management's request to implement the quality policy of the firm. It is a knowledgecreation process based on a scientifically designed dialogue with empirical facts, using statistical methods. The statistical approach entails epistemological specificities of the knowledge-production process, which will be explained later, 
providing a description of the first factor. The second factor, i.e. the integration of managerial demands, is responsible for the originality and the ingenuity of the methods designed in this instance, as compared with the methods used in academic settings. A major distinction is found in the business criteria of efficiency and limitation of resources. In a firm, knowledge production must in general obey certain restrictions in cost and deadlines. It must also respect the organization of the work of industrial production without upsetting it, and it must be adapted to the skills of the industrial production personnel which are not as high as those of scientific research technicians. SQC takes intimately into account such requirements that are important for management.

The dialogue will be used as a metaphor to describe the knowledge-creation process, constrasting with the more academic language of hypothesis testing and planned experiment. We argue that an interactive relation is established between theory and facts, which we represent as a dialogue. This is a very versatile notion and our intention is not to develop a full theory of it. We are interested mainly in the formal aspects of the dialogue as a kind of conversational exchange. First, it is a conversation between two persons or entities, without the intervention of a narrator. Second, dialogue is governed by some rules of "turn-taking in conversation" (Sacks et al., 1974) which constitute a distinctive feature and takes part in the narrative function. Third, a dialogue is not pre-conceived or precisely planned, it may lead to unexpected matters or ideas as an exploration does.

The very idea of a dialogue stems from the observation that SQC is comparable to a monitoring system, where the production process is frequently questioned by the men in charge of its management. More ambitiously, as we shall see, SQC is designed not only to ensure that everything is in order with the production process, but also to raise new hypotheses about unexpected sources of variation in this same process (this is known as "continuous improvement" in the Total Quality Management movement). This approach is sequential, exploratory, each step bringing incremental knowledge in a mode closer to dialogue than to planned experiment.

The paper proceeds with a first part dedicated to a general presentation of the case in its historical context and to a deeper analysis of the knowledge involved, in order to explain the metaphor of the dialogue. The two following parts will show how the managerial demands have been incorporated in the SQC theory and tools. They deal 
first with the timing and rhythm of the dialogue, and second with the economic aspects of the procedures for observation and decision making. The aspect of the workforce capabilities will only be tackled along the way, for a thorough treatment would require much longer developments than the present article can offer.

Our approach is quite different than the debate about "the relevance gap in management research", which raised many questions about the nature of knowledge created by research at the interface between business and academia (Starkey \& Madan, 2001). In the example studied here, there is no visible "interface" between academia and business: the innovative methods originated inside the firm, with very little intervention of academics. We are thus interested in knowledge produced inside the firm, about some empirical objects, with the aim of contributing to some definite management problems. Besides, we are not concerned by disciplinary distinctions between, for example, management and engineering knowledge. Readers might feel that SQC belongs to the field of engineering or statistics, not management. But we follow Starkey \& Madan's opinion: " Knowledge production has shifted towards interdisciplinary research in the context of application, problem-solving and greater collaboration ". The crucial point is how knowledge creation methods, tools and practice, in any field of activity of the firm, are in tune with managerial goals and orientations. Using the notion of "fundamentals" put forward by March (as quoted in (Starkey \& Madan, 2001)) and (Weick, 2001), we consider our work as an attempt at characterizing the fundamentals of SQC, considered as a double-sided activity articulating scientific and managerial perspectives. In our opinion, an analog approach could be applied to other fields of activity inside the firm, provided that empirically-grounded knowledge matters.

\section{MATHEMATICAL STATISTICS, A THEORY FOR QUALITY CONTROL}

Numerous accounts of the history of statistical quality control help us reconstitute the origin of these methods (Dodge, 1970), (Fagen, 1975), (Bayart, 1996), (Juran, 1997), (Bayart, 2000). In the 1920s, different statistical methods emerged at the same time in several industrialized countries (United States, France, Germany, Great Britain). That these methods appeared independently suggests that it is not just a question of the simple spreading of an innovation, but a deeper phenomenon. The most probable common link is the development of the mass production of technical items defined 
by precise and measurable technical characteristics, such as automobiles, telephones, armaments, and electrical equipment.

\section{Statistical control in the industrial policy of AT\&T}

At AT\&T in the United States, systematic research and development actions had been conducted from 1922 in order to find scientific remedies to the problem of unsatisfying quality of the products. Over several years, researchers produced a corpus of theories and operational methods which will be the main example on which this paper is based. First, then, a few words about the historical evolution of the question of quality control in this firm.

Around 1890 the AT\&T Company committed itself to "quality assurance", in which they guaranteed customers the sound quality of products supplied (Fagen, 1975). The main means to quality was at the time inspection, the physical examination of products to see if they conformed to specifications. Products were inspected several times before being delivered, and these inspections needed a considerable number of staff. Nevertheless, customers complained of defects in the products supplied, instituted legal proceedings, and inspected the deliveries once again intending to make the producer pay for these expenses. Thus inspections (and costs) were multiplied at the initiative of different players.

Numerous procedures were established in order to rationalize inspection and reduce costs. Different types of organization and responsibility were also tried. But the problems persisted - the level of quality remained insufficient as regards specifications. Control by inspection obviously needed a theoretical and practical reform. In 1922-23, management decided to develop a more scientific approach to the problem. Within the Engineering Department of Western Electric they created an engineering department for the inspection, which was transferred in 1925 to the newly created Bell Telephone Laboratories. The historian of the Bell Labs (Fagen, 1975) noted the first systematic set-up of Quality assurance in the industry.

The Bell Labs were in fact an exceptional place for the development of such a type of research, mixing scientific and industrial approaches. The scientists in charge of quality research enjoyed a large autonomy to investigate the industrial aspects of the problem as seen by the various players. The Inspection Engineering Department formed in the year 1923 inside the Western Electric Engineering Department had the responsability of taking a "Company-wide view", including a critical analysis of the 
work of design (Fagen 1975, p.861). For example, this gave the scientists the freedom to experiment with new methods in real situations (Dodge, 1969). This raised many discussions with the various potential users, pros and cons. The legitimacy of the scientific approach supported by the Bell Labs potential and prestige probably had the effect that the new method was not rejected but adapted through negotiation of its characteristics. A comparison with the other firms or institutions where similar problems were tackled in other countries leads to the conclusion that the links established between the Bell Labs scientists and real industrial situations within the firm were a decisive factor in the reputation gained by the AT\&T statistical methods. It may be added that the Hawthorne plant, where the quality scientists often made observations, was at that time a place of many experiments in various domains of knowledge, including the social sciences. At AT\&T, science was not alien to the world of industry.

Two aspects of the quality research are particularly interesting for our point. The first consisted in studying the acceptance sampling, i.e. the sampling of lots in order to obtain a statistical assessment of the quality of the lots delivered to the consumer. The man in charge was Harold F. Dodge (1893-1976), an engineer from MIT. The second mission, entrusted to the physicist Walter A. Shewhart (1891-1967), concerned the control of the quality of products at manufacture process level. Shewhart was to perfect the method called "the control chart," which skillfully uses the graphic diagrams to give an image of the quality of the production. Above all he developed a new theory (the "constant systems of chance causes") to help improve the regularity of the manufacturing process by detecting and eliminating the unexpected causes of variations in the properties of products. These two closely complementary approaches used the resources of mathematical statistics which were at the time under development.

\section{In acceptance control, new methods for an old problem}

The main problem with inspection is due to a production too great in number to be checked in totality ${ }^{1}$. Then it becomes mandatory to check only part of it, but in what proportion ? The practice of taking a sample to evaluate the quality of a delivery is

\footnotetext{
${ }^{1}$ Or when inspection is impossible without destroying the part. This is the case for ammunitions or fuses of shells and it has been studied early by military engineers and gunners.
} 
quite ancient ${ }^{2}$, but the sample sizes traditionally used were much too small to yield reliable results. Common sense usually underestimates the size needed for a sample to be representative of the whole. It is not the least contribution of probabilistic theory to mitigate common sense and to allow for rational calculation of the risks of error as a function of the sample size. It shows why the old practices did not lead to satisfactory protection for producer or consumer.

Thus, the knowledge to be produced concerns the proportion of defective items in a lot of products contractually delivered by the producer to the consumer. But what are the "facts" to be considered in the organization of a dialogue ? The operational procedure prescribes to take a sample of a given size, to test its parts and, depending on the number of defects found, to make a decision of accepting or rejecting the whole lot. In the methods designed at Bell Labs, a rejected lot had to be screened in order to replace the bad elements by good ones. The theoretical work was dedicated to the formulation and study of the mathematical relations between the various parameters. Many different ways of taking the sample were imagined and studied. As we shall see later on, those constitute different modes of organizing the dialogue with the facts.

In statistics vocabulary, this is the question of testing a hypothesis (the level of quality of a population of products) by sampling. At the time, this problem led to relatively simple probability reasonings, on the model of the drawing from an urn of balls of two colours (good/bad), but entailing heavy calculations. The lot is represented by the urn, whose exact make-up is unknown, and the sampling by the balls which are drawn out. The novelty contributed by the theory lies more in the formalization of risks of error associated with the decision-making. The consumer's risk is defined as the risk of accepting as correct a lot of insufficient quality. The producer's risk is that symmetrically a good lot is deemed insufficient in quality ${ }^{3}$.

By contrast, Frederick W. Taylor may be taken as an example of a non-probabilist approach when he sets out a theory of the control organization of steel balls intended for bicycle bearings (Taylor, 1911). A team of women inspectors examined all the

\footnotetext{
${ }^{2}$ Coins were checked by sampling as early as the Middle Ages (Stigler, 1977); sampling practices developed notably with the railway industry in order to check the rails and various safety parts, not to mention the armament industries.

${ }^{3}$ These two risks correspond to the type I and II errors in the classical statistics terminology of Neyman-Pearson, which was published only in 1928 (Neyman \& Pearson, 1928).
} 
balls manufactured in order to eliminate the faulty parts, but inevitably they made errors. For Taylor, the tendency to errors is a property of each person, which he called the "personal coefficient". In order to determine this coefficient, Taylor instigated an "over-inspector" who checked the sorting made by each inspector. In the logic of his principles, Taylor organized the selection of the best inspectors, those who made the least mistakes. The arrangement thus worked at two levels - it sorted not only the balls but also the inspectors, who were treated here as objects of study, as technical devices with inherent technical characteristic.

In Taylor's model, the inspection error appears as a certain percentage of the number of parts examined. The quality defects are not entirely eliminated and the theory does not suggest any practical way to treat them, as this is a determinist model. The statistical models on the other hand choose the number of defects as a scientific object, consider it as a random variable and submit it to calculation. Statistics enable calculating the size of the sample to be checked when one fixes the level of risk of accepting a certain percentage of defective parts (consumer's risk). It offers a new way to assess theoretically the efficiency of the control.

\section{The process control, new theoretical framework based on a non-determinist epistemology}

While the preceding form of control uses an intuitive and conceptually simple model, the probabilist model of drawing out of an urn, the scientific hypotheses supporting the process control approach are far more radical. Indeed, they challenge the determinist nature of physical phenomena, including those exploited by the industrial processes, thereby undermining many traditional knowledge foundations. Shewhart, a PhD in physics (1917), endorses the epistemology of statistical physics by proposing that all the physical quantities are of a statistical type. For example his first article published in the scientific journal of AT\&T, the Bell System Technical Journal, states abruptly in its very first sentence:

"We ordinarily think of the physical and engineering sciences as being exact. In a majority of physical measurements this is practically true. (...) We are led to conceive of the physico-chemical laws as a statistical determinism to which 'the law of great numbers' imparts the appearance of infinite precision" (Shewhart, 1924) 
Even when the physical quantities appear to us to be measurable as accurately as one wishes, all one sees is the expression of the law of large numbers. The variability of the physical quantities is at the very heart of reality. What consequences does this new epistemology bear for the knowledge-creation processes in industry? To understand this, we must go into some technical explanations.

To argue in favour of statistics, Shewhart chooses a case of great importance to the company, as it concerns fundamental aspects of the quality of the microphone of Bell telephones. These used a capsule containing thousands of granules of carbon, which could stop the microphone working correctly in certain positions (Fagen, 1975). The electric resistance of the capsule is a crucial characteristic of the device, but it varies in many unexplained ways:

"In practice it is necessary to know why slight changes in the manufacturing process cause large variations in the resistance characteristics of the carbon. The same process that improves one microphonic property may prove a detriment to another. It is in the solution of some of these problems that statistical methods have been found to be of great value in the interpretation of the results." (Shewhart, 1924)

According to this scientific opinion, it is impossible to manufacture two products exactly alike. Industry must therefore adapt to the variability of phenomena. Luckily, this variability is often limited and may be expressed by statistical laws. This is not the case for all the phenomena but, when these laws exist, a suitable line of study must facilitate their identification. The fundamental question of statistical control thus becomes: when should variations observed in the product quality "be left to chance", or be acted upon in order to reduce them ? (Shewhart, 1931). This question receives an answer in terms of "control limits" established by means of mathematical statistics, as will be explained below.

The variability of the product is the result of numerous factors some of which can be identified and eliminated. Shewhart therefore suggested a method for identifying possible causes of variability which can be eliminated. Such identifiable factors are called "assignable causes of variation". The causes not assignable are usually of very small individual effect and, as they are numerous, they add up to the effect of "the law of large numbers". As mentioned in the quotation above, this allows a stability of the variable based on statistical laws. A manufacturing process is referred to as "under statistical control" when all the assignable causes of variability have been eliminated. In these conditions, the law of large numbers applies, and the physical 
quantity follows a stable statistical distribution. In particular, with a given probability, the values will keep between two limits, one upper and one lower, that can be computed from the observed facts. In ordinary practice, the limits situated at three times the standard deviation on each side of the arithmetic mean are chosen as "the control limits" ${ }^{4}$. Shewhart underlines that a system does not allow valid predictions regarding its output unless it is under statistical control. The usual statistical methods for the identification of statistical distributions from empirical data provide means to estimate the numerical parameters useful for quality control.

The defects of manufactured products are, in this view, the result of poor suitability between the variability of the physical processes involved in the production and the specifications that the product must meet. It is possible that the specifications are too narrow for the spontaneous variability of the process and the product. In this case, there is no other way but to sort the products and to eliminate those that are not in compliance.

As early as 1924, a graphical tool was designed to show at the same time the observed value of the variable and the limits that its variations should not exceed ("the control limits"). This "graphical report", as Shewhart called it, was to become within a few years the well-known "control chart", when it allowed for a quick follow-up of production and a more detailed surveillance of the variability (see fig. 1).

The procedure to be followed in order to establish the control chart can be summed up this way :

1) consider the production process over a certain period of time, determine the statistical distribution of the characteristic to be controlled;

2) calculate the control limits, situated at 3 times the standard deviation of the distribution observed;

3) in the standard form of the control chart (see fig. 1), chart the two indicators of the statistical distribution of the characteristic to be observed : the mean value (or arithmetic mean) and the dispersion (standard deviation or range) -- diagram with control limits has to be established for each indicator;

\footnotetext{
${ }^{4}$ We shall explain more precisely in $\$ 3$ the origin of the "3 sigma rule" in connection with economic reasoning. This rule means that, with probability .997 , a correct decision occurs if a variation falling outside the control limits is taken as an indication of a significant cause of variability.
} 
4) continue to observe production by taking a sample at regular intervals; for each sample, calculate the values of the indicators (mean value, dispersion) and plot them as points on the corresponding diagrams; continue as long as those points remain within the control limits;

5) if a point falls outside the control limits, it is advisable to look for an "assignable cause of variation" (this will be commented below).

We have thus described the fundamentals of the scientific epistemology which is drawn upon in SQC. The procedure just described implies, when put to practice, a subtle interplay of models and observable facts, which we will now explore using the dialogue metaphor.

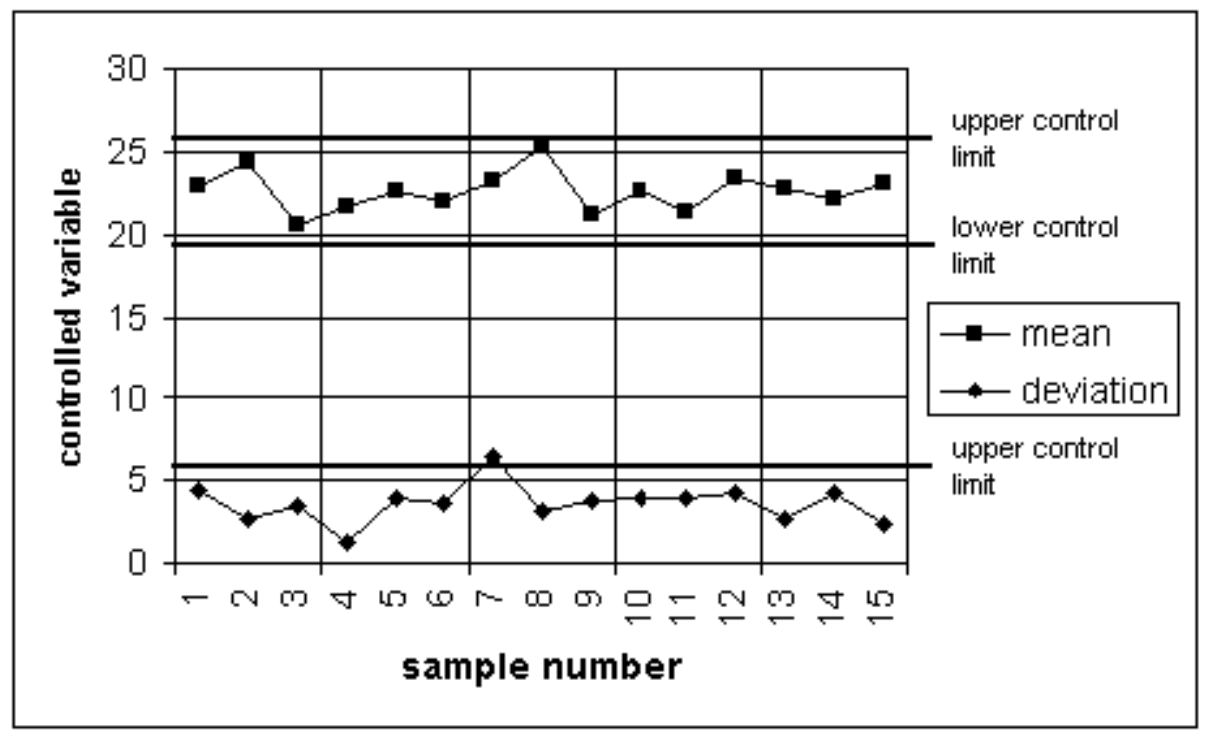

Fig. 1 - a standard control chart, in average and range

\section{The modes of "dialogue with the facts"}

The statistical theory of SQC has an ambiguous relation with the facts, i.e. the variability of the characteristics of manufactured products. The basic question put by Shewhart is "how much variation should be left to chance?". One could expect an answer coming from some norm, or from the client's needs. This would probably be enough on commercial grounds. But such an answer did not satisfy Shewhart, he wanted the answer to come from the very facts under study. He repeatedly 
questioned the facts to reveal the assignable causes one after the other. That process of chasing the assignable causes stops only when no cause remains that can be identified (an economic viewpoint should also be considered, as we shall see later). It stops when the production process has reached a state of statistical control.

Such an observation encourages us to distinguish two modes of questioning the facts. The first mode assumes the process already placed under control. The facts then correspond to a monitoring of the production process. In this situation, the challenge is to establish that the product characteristics have not varied beyond set limits. Here, the dialogue is situated in a fixed framework, for example a statistical model of the production, to which a prescriptive value is given. This model is manifest by the control limits shown on the control chart. As long as the representative production points remain within these limits, the situation is compatible with the model and one need not intervene. Intervention is even inadvisable as this would only increase the variability.

In this mode, the dialogue is limited to ensuring the situation remains in agreement with the model. Hence we shall call it normative. In case of disagreement, the decision to be taken depends on the status of the model. If the model represents the expected specifications of the product, it constitutes a standard of exogenous origin, and in principle cannot be affected by the facts observed. Then the conclusion is that the product does not fit the specifications. If on the contrary the model represents the spontaneous variability of the phenomenon under control, a significant variation indicates then that the model has ceased to be valid. Such circumstances are taken care of with a dialogue of mode 2.

The second mode of dialogue between facts and theory occurs where one aims at bringing the production process under statistical control. A process out of control produces an output which cannot be represented by a statistical model. It is not even possible to predict with some precision what the output will be because the causes of variation are not known. The goal of the approach is not to learn more precisely the laws governing these causes of variation but to reveal and eliminate them-at least the causes exerting an identifiable effect, the so-called "assignable causes". This mode of dialogue will be called exploratory.

In this case the control chart is used with an intention opposite to the one in the first mode: to show the assignable causes of variation. To bring the system to a state of statistical control, one starts by observing production and establishing an initial 
model of the "control chart" type. This comprises control limits which in turn help reveal significant variations which then give rise to an inquiry and to the formulation of a hypothesis concerning the variation causes. The hypothesis is tried out through a physical modification of the production system. The production is once again observed, a new model is established. In other words the control limits are recalculated. If this new model does not reveal significant variation, one can stop there - if not, the procedure is iterated again and again.

The state of statistical control is an asymptotic state, reached when it appears no longer useful to change anything at all in the production system. The progression towards this state is made by the sequence of elementary cycles comprising three steps named : "specification", "production" and "inspection". Specification is the step of prevision: if the process is under control, one is able to predict, at least statistically, its output. If it is not under control, the specification will usually not be valid. Production is the step of the physical action. Inspection is the step where one sees if the prediction holds. If not, it means that the system is not under control. On the basis of engineering knowledge, a change has to be made in the physical state of the system. A new cycle can then start.

These three steps are, for Shewhart, comparable to the three steps of the experimental scientific approach:

"It may be helpful to think of the three steps in the mass production process as steps in the scientific method. In this sense, specification, production, and inspection correspond respectively to making a hypothesis, carrying out an experiment, and testing the hypothesis. The three steps constitute a dynamic scientific process of acquiring knowledge." (Shewhart, 1939). 


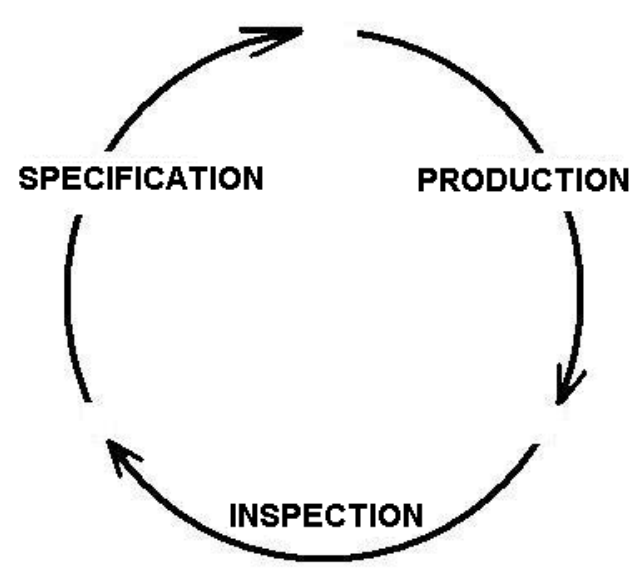

Fig. 2 - The Shewhart cycle (after Shewhart, 1939)

\section{Specificities of the knowledge produced}

The knowledge produced along this iterative process appears to be of a particular kind which, following (Knorr Cetina, 1999), could be called "negative", referring to the knowledge developed by high-energy physicists as they try to separate the events really produced by a particle collision from all the noise produced by the experimental equipment. Shewhart does not try to exhibit positive laws governing the trends observed in the product characteristics, he does not study them per se but only in order to eliminate them. His goal is to eliminate all notable sources of perturbation on the production process, leaving only those which produce random effects. Meanwhile he interestingly underlines the difficulty of establishing a random system within the context of a firm: "in mass production the statistician has learned by experience that random effects do not just happen, even by careful planning. If the industrial statistician ignores this fact and makes predictions as if he were dealing with randomness, he may expect many of his predictions to go wild." (Shewhart, 1939, 47). This remark nicely catches the paradox or oxymoron that resides in the project of a "planned randomness", yet a condition necessary to the statistician's work.

Another pecularity of this knowledge is the difficulty of proving that a system is under control: again it is a negative property. It cannot be positively established but only refuted. It is a hypothesis valid for an unknown duration, until it appears invalid. Even in the most favorable conditions for controlling a system, the laws of statistical sampling require quite a large sample-more than one thousand pieces, says Shewhart-to allow a reliable conclusion stating the absence of assignable causes. 
The dialogue thus appears as a form of questioning the empirical which is justified by the sequential character of the elimination of the assignable causes, one after the other. Indeed a major cause of variation has to be removed first so that the other less important causes can be detected. The exploratory character of the sequence is also well represented by the dialogue metaphor: one does not know ex ante what factor will appear as a cause of variation. One does not know where the dialogue will lead. Even if one has some hints about the important factors, there is no proof until the dialogue has reached the corresponding development and the physical change has been tried in the process. This is a sequential exploration, where the explored space is not pre-defined but opens out during the exploration itself.

The progression towards the goal of a random system is composed of a succession of trial-and-misses. The identification of causes is part of the knowledge creating process but only with the intention to remove them. The dialogue metaphor underlines that the practical result is obtained only at the end of a succession of exchanges, which stops when there is nothing more to be told. A single loop of the cycle described above (which is known as "the Shewhart cycle" ${ }^{4}$ ) is of no interest unless it is followed by as many others as will be necessary to exhaust the matter.

Let us now summarize our conclusions concerning the nature of knowledge in the case of SQC.

1) More than for most other sciences, it is accepted that statistical facts are not directly perceptible by our senses and are constructed by means of theory. Our spontaneous intuition of the statistical facts, notably of sampling, is usually misleading (as is shown by the common under-estimation of the size of the samples to be controlled). This entails a systematic recourse to procedures to construct the facts and make them ready to use. Thus there are procedures for selecting the sample, copying out the data, leading the calculation, representing the results, etc. - as shown in (A.S.T.M., 1933). The facts themselves are abstract and their usability depends upon their representation.

2) The representation of the facts draws upon various modes: numerical (sheets of data, lists of measures...), graphical (representation of statistical distributions,

\footnotetext{
${ }^{4}$ W.E. Deming popularized a version with four phases, known as "Deming wheel", or "PDCA" for Plan-Do-Check-Act. The added phase is "Act", which is the implementation of a physical change in the production process, as a consequence of the conclusion drawn from phases 1 to 3 - and eventually to prepare a next cycle.
} 
histograms...), symbolical (equations, formulas...). Statisticians devised a great diversity of representations for statistical data (Tufte, 2001), (Tukey, 1977). Representations can be designed differently according to their destination: exhibit results, take a decision, act upon the production process, etc. Some are polyvalent: thus, the control chart is at the same time a decision tool, a representation of past production, a representation of the normative statistical model of the product (control limits).

3) The production of knowledge aims at eliminating the causes of variations in the production process, not at learning about those causes per se. It is of course necessary to gather knowledge about the causes in order to eliminate them, but the nature and the amount of knowledge to build is not pre-determined and appears in the flow of the interaction with the facts. For example, it might appear necessary to build very precise knowledge concerning a permanent factor such as ambiant air temperature or hygrometry.

4) Random events gain recognition as events to be managed in accordance with some definite rules or laws. They have to be recognized as an inevitable part of the production events (Deming, 1986). More precisely, the state of statistical control, which is a necessary condition to make valid predictions on the outcome of the production process, may be destroyed by untimely interventions on that process.

5) The metaphor of the dialogue captures more adequately than the traditional view of planned experiments and hypothesis testing the sequential character of the approach prescribed to reach a state of statistical control. The notions of sequential exploration and incremental knowledge become important in the view of instituting a process for acquiring knowledge in order to control and monitor the production process.

We shall now review successively the topics of time rhythms and costs to see how the management expectations are articulated with the peculiarities of the knowledge by means of the empirical machinery.

\section{CONTROL RHYTHMS}

The temporal rhythms of the control are a central feature in our dialogue vision of the process of knowledge acquisition. Overall, the main point concerns the rhythms of the dialogue with the facts, and notably the rhythm of the observations and the conclusions drawn. (Sacks et al., 1974) introduced "turn-taking" and its organization 
as an object of study in conversation analysis. Recognizing it further as "a prominent type of social organization", they note that "for socially organized activities, the presence of 'turns' suggests an economy, with turns for something being valued-and with means for allocating them, which affect their relative distribution, as in economies." The notion of "turn-taking system" suggests that turn-taking is not arbitrary but must follow some rules, both social and technical. A point of interest is that the activity submitted to turn-taking may be "adapted to, or constrained by, the particular form of turn-taking system which operates on it" (ibid.). We argue then that the organization of turn-taking between theory and facts is a crucial feature of a knowledge-creation process. We will be concerned in this section with its temporal dimension, in the following section with its economic aspects.

We can approach the question from three different angles - the rhythm of phenomena to be observed, the rhythm required for the actions on the system, and the time "off line", separate from the main activity.

The first time scale considered concerns the rhythm of phenomena inasmuch as they follow the laws of nature, a rhythm which is imposed on the observers and which must basically be taken into account to conceive any system of knowledge creation. This is well expressed by W.E Deming:

"When machines are turning out pieceparts by the thousands or even millions monthly, the industrial statistician does not have to wait long to see his predictions tested out. In agriculture, years are often required--a crop must be sowed and harvested again and again until the evidence is definitely for or against the prediction that one treatment is actually better than another." (Foreword to (Shewhart, 1939))

This remark goes to the point that the facts-theory dialogue in industrial settings is much more revealing by unit of time than in agriculture. The epistemic culture in agriculture rests much more on hypothesis testing and planned experiments than on dialogue. The methods used by the various scientific disciplines usually reflect in some way the rhythms inherent in their relation to the empirical world and to the facts. Nuclear high-energy physics, for example, typically leads an experiment with real empirical facts (i.e. implying particle colliders) for a few months every five or more years (Knorr Cetina, 1999). The scientists thus gather data on rare occasions and meanwhile spend most of their efforts on data analysis. 
We suggest that an intensive dialogue with the facts favors exploratory enquiries in a sequential manner, as one quickly gets an answer to a question posed. The answer suggests another question, which can be put to test. A fast dialogue gives numerous opportunities to try out various means and to improve them by repeated trial and error. Inversely, in the fields where experimental feedback takes a long time, theory is turned onto making more effective experimental work to obtain a better output from fewer observations.

Advances in the overall knowledge appear here as the iteration of elementary cycles of knowledge acquisition (Shewhart cycles) endowed with their own time duration. We need to remember that industrial knowledge is linked to action, which has its own temporalities. These two orientations often pull in opposed directions, notably concerning time limits, and this may perturb knowledge acquisition. Hence the importance of "the epistemic machinery" when it succeeds in settling compromises through embodiment in material artefacts or organizational routines. This aspect of the temporality inherent to the phenomena under study therefore defines a very general frame within which all sorts of micro-temporalities are built and embodied, bringing together observation, knowledge and action.

Obviously the production process has its own temporalities, as well as the devices for observation and production of knowledge. The rhythm for the return of control information must correspond to the production rhythm and to the speed of decisions to be taken for the monitoring (which can still be different). This is the second time scale, to consider in conjunction with the preceding. There exist of course strong ties between the two, but one might imagine the second more flexible than the first, as it results partly from management decisions.

It was thus recognized after a few years of experimentation that small samples taken at shorter time intervals provide an image of quality sufficient for the purpose of monitoring the production process. Indeed, such an image is less precise than the one obtained with large samples, but the dynamic view it allows is a great advantage. Thus Shewhart expanded the statistical theory of small samples and tested its applicability to many different conditions. It is to be noted that the initial impetus of small samples theory was given around 1900 by Student, another statistician working as an engineer in industry. In disagreement with the statisticians of his time who worked in agronomics, he could not collect series of data long enough for him to apply the usual methods, because it would have disturbed the production process. 
This example shows the importance of a fit between the empirical machinery of the knowledge production process and the setting of the production process.

Finally, a third time scale arises from the work outside the operation situation, in frameworks built "beside" the real process, for example for the training of personnel, to make tests, simulations, or repetitions. The temporality is no longer imposed by the industrial process itself, but built by the investigators or instructors depending on their specific objectives. These situations are characterized by their autonomy vis-àvis the process to be studied, which is however represented by certain devices, symbolic systems (mathematical models, for example) or equipment (mock-ups, simulators). In his "Frame Analysis", the sociologist E. Goffman calls such situations whose meaning derives from another situation taken as a reference, and which constitute a transformation of this original situation, "keyed" (Goffman, 1974).

The dialogue with the facts can then question multiple combinations of factors of all kinds, ranging from abstract representations by ways of mathematical models to the behavior of real persons confronted with experiments or training practice. While the role of mathematical models has been much studied in the organizational literature, the role of the simulations is less often analysed.

Simulation tools and models are important constituants of the epistemic culture of any knowledge field. In the case of SQC, models simulating "constant systems of chance causes" were most useful to experimenting with sampling procedures and finding the most efficient sample size. They were constituted by a salad bowl containing tokens marked with figures, from which random draws were made (Shewhart, 1939, 165, 183). This device clearly belongs to the "empirical machinery". It reproduces a world of random events governed by a fixed and stable law of probability, with which all dialogue conditions can be freely experimented. In industry, simulation is most frequently used for training purpose.

The temporal dimension is fundamental to control operations as soon as the control intends to institute a follow-up of the controlled process, yet more if it implies monitoring of the process (i.e. acting back on the process). The dialogue metaphor is well-suited to the periodic observation or interrogation of the process. It records and represents a history of the qualitative performance of the production process, which may be seen as growing knowledge on the process. Also supporting the metaphor is the fact that frequent interrogation, although not very precise, is preferable to a more accurate picture obtained less frequently. In conclusion, it is 
important to emphasize the conjunction of the different time periods of the process studied, of the observation means and of the decision process. The empirical machinery of the knowledge creating process often bears its own temporal characteristics, which may be different from those embodied in management tools in use.

\section{THE COST OF INFORMATION AND KNOWLEDGE}

We shall now examine how the economic dimension is taken into account in the tools and methods of statistical quality control, as well as in the organization of the facts/theory dialogue. The general perspective is to balance the costs and value of knowledge acquisition, advantages.. Statistical quality control clearly showed that acquiring information has a price and provided means for confronting these issues. Among the costs to consider, special attention was given to the direct costs of control operations. The cost of labour could be very high if qualified workers were necessary. But the intuitive meaning of control charts, for example, made them usable, after some training, by workers on machine understanding and applying elementary arithmetical operations. In industry, cognitive tools associated with ingenious graphical designs are frequently used for bringing sophisticated scientific or technical methods within the reach of ordinary workers.

An important contribution of theoretical statistical thinking concerned the efficiency of sampling in relation to its cost. Let us recall how important the cost of inspection was in the early days of quality control, reflected in the conflicts about who should pay for it. The "cost of inspection" is supposed to be directly proportional to the number of inspected elements. Thus, the theoretical question was to minimize this quantity, under the constraint of a given level of protection for the client (the client's risk being the risk of accepting a lot of insufficient quality).

An economic view of the dialogue occurs when it is shown by theoretical thinking that repeated samplings and trials are more efficient than a "single shot". The (theoretically) most efficient method, called "sequential sampling", prescribes to take samples of one piece at a time. As we shall see, however, it does not fit well to practice, mainly because the dialogue with the facts may take a long time to reach a conclusion, whereas each speech turn appears too costly in comparison with its contribution. 
Regarding the control chart, the economic viewpoint concerns the balance between the two classical types of statistical risks of making a bad decision. The proposed approach mixes theoretical and empirical considerations, leading to an "optimal" position of the control limits, neither too loose nor too tight. The control chart also implies some conventions that make possible a dialogue with facts gathered in other firms or industries, these conventions being supported by institutions for standardization.

\section{Minimizing the inspection costs with a warranted level of protection}

Dodge and Romig offer to determine the "most economic" plan of inspection - the optimum being where the plan, for a determined consumer risk, minimizes the average number of parts examined, and thus the cost of inspection. There are many plans satisfying the condition of a given producer's risk (which is the risk of rejecting a good lot), and the selection of the most economical one implies a huge amount of calculation. This could be done thanks to the technical resources of Bell Labs but was not within the capacities of an ordinary firm. The numerical results were kept as a professional secret for many years (Juran, 1997) but the calculation principles were published in 1929, likely with the intention of promoting the reputation of AT\&T on the topic of quality assurance.

When at last the numerical results were published (Dodge \& Romig, 1944), it was in the circumstances of World War II under pressure of the Government who wanted them to be implemented in the armament industries. The publication takes the form of a collection of numerical tables where every case and answer is "precomputed"- to use a modern expression (Norman, 1993).

Here the dialogue is not to be found in any single use of the numerical tables but in their repeated use to support a regular consumer-producer relationship. That is where economics play another part: the contractual conditions besides the technical aspect of sampling state that the sampling costs are to be paid by the producer and that any rejected lot shall be inspected entirely, in order to replace the defective parts by good ones. This way, the consumer receives a lot of perfect quality while the producer's expenses increase. On a regular basis, such dispositions set economic incentives on the producer, driving him to maintain the quality level specified in the contract. 


\section{The sequential sampling, an optimal solution in theory but not in practice}

The question of minimizing the size of the sample had important developments in academic circles after the Second World War. Sequential sampling however dates from the war itself, as it was proposed by statisticians working for the U.S. Government, explicitly in order to perfect economical inspection methods (Klein, 2000). The novelty of this method is that instead of fixing a priori the size of the sample and drawing all the elements in one go, they are drawn one by one and after each draw, a decision rule prescribes to accept the lot, or reject it, or draw another element. The size of the sample is then a random variable, and it is proven by theoretical means that one obtains statistically in this way the smallest samples possible for a given consumer's risk. The dialogue with the facts in this method is made up of the most elementary units possible, but the number of exchanges is random and can be very large.

However the sequential analysis was not considered as optimal in practice, in spite of economical performances considerably better than the existing methods. It was not very successful with the inspectors. Dodge (1969) noted that the inspectors at Western Electric were opposed "to a plan that can't make up its mind" - the draw can in fact continue for a long time. This sounds exactly as if the inspectors found such a conversation with the facts too tiresome for the result. The obligation to draw the elements one after the other was moreover fairly difficult to implement. To draw the samples required handling (open the packaging, undo stacks of boxes, etc.) and it is far quicker or less expensive to take all the parts at once. Sequential sampling, then, seems to generate an endless dialogue with too little information at each "speech turn".

The favorite method of the inspectors was in fact a two-step dialogue with the inspected lot. It is called "the double sampling", and it "gives the lot a second chance" (Dodge). Set out in an intuitive manner, the approach is as follows - an initial sample is drawn; if it is very good, the lot is accepted; if it is very bad, the lot is refused; if it is between the two, a second sample is drawn which will answer the question. An inspector usually does not like to refuse a lot as this creates tension with the manufacturing departments; if he has to refuse it, he wants sound reasons to do 
so, and two successive failures to the test seem to him sufficient proof ${ }^{5}$. Such a procedure is more economical than single sampling. The statisticians of the Bell Labs developed it as soon as the 1920 's, without the sophisticated mathematical methods invented by Wald, but with a lot of computational resources instead. All the parameters can be computed so that the procedure ensures a given level of protection and tables of numerical values to be used have been built up, becoming an essential part of the empirical machinery.

\section{The economic reasoning applied to the search for causes of variability.}

The control chart offers another aspect of the economic reasoning : finding a reasonable balance between two opposite risks of making a poor decision. Cost considerations lead to decision rules supporting statistically optimal strategies. The control chart being a purely statistical tool, it indicates a variation too large to result from a state of statistical control but it does not identify the cause of the variation. Such a task requires often costly empirical investigation. These investigations are economically justified only if the expected return is higher than the cost. Hence, two kinds of risks must be considered from an economic viewpoint: the risk of "false alarm", associated with the cost of an inquiry without a valuable outcome, and the risk of "no alarm", whereas it would be justified, with a cost ensuing from insufficient quality.

The calculation of the control limits, as explained by Shewhart in 1931, proposes a solution to this question. First of all he shows that there does exist an economically optimal position of control limits - if they are too loose, they do not detect variations adequately, the quality of the product is not controlled as well as it could be; if they are very tight, there are too many false alarms and also losses. It was thus clear that there could be an optimal position, but it was not clear where it might be.

Finding this position through calculation is not possible in general because the statistical distribution of the quantity studied is not known. In this case, symbolic equations are essentially a medium of mathematical abstract reasoning. To establish the numerical values of coefficients, Shewhart relies on the pragmatist epistemology derived from C.S. Peirce's philosophy. In Shewhart's approach, theory provides the

\footnotetext{
${ }^{5}$ In the thirties, out of the thousands of sampling plans used at the Hawthorne factory, about $70 \%$ were double, 25\% single, and less than 5\% multiple (Dodge, 1969).
} 
form of the proposal, and experience provides the numerical coefficients that the theory does not allow us to calculate (Lovitt, 1997). We may acknowledge here Shewhart's initial training as an experimental physicist.

This experimental point of view is reflected in a rather surprising manner by an uniform rule for all the situations. One would have expected the control limits to be adapted to the precise situation where they should be used. But Shewhart argues that they must be fixed in any case at $3 \sigma$ (standard deviations) on either side of the average of the distribution, which corresponds to a very small risk of a false alarm. ${ }^{6}$

Above all, he puts forward the need to simplify and standardize industrial practices in the use of the control chart tool. The compromise on the standard "three sigma" results from a point of view of industrial standard-maker - these products are intended to be exchanged in the industrial networks and it is essential that the measurement criteria be standardized. One finds several contradictions and compromises here which express the tensions between the prescriptiveness of the approach (deciding a priori of "how much variability is admissible" in general) and the preoccupation of establishing this standard on the observation of facts (deciding "how much variability is admissible for a specific production process").

\section{An institutional level of dialogue with the facts}

This leads us to identify a third mode of dialogue, not with the facts originated in the process studied but with those to be found in other industries or firms, that have to be compared with each other or fit together in some way, according to the needs of industrial and commercial networks. This amounts to defining some criteria of equivalence (Boltanski and Thévenot, 1991) with their means of operationalization. Shewhart and Dodge dedicated much effort to work in committees with engineering associations acting as normalization agencies in the United States, such as the American Society for Testing Materials. This was clearly strategic action to make their ideas known and to help with the diffusion of standards implemented by AT\&T. But standards are shared conventions and must necessarily compromise over the singularities of their multiple applications. Thus, a knowledge acquisition process designed very accurately to fit with a specific production process is at the end overdetermined by some general conventions which regulate the exchanges of products. 
The dialogue with institutions in charge of standardization has been of particular importance for the quality control movement. The British Standards Institution, for example, recognized Shewhart's works, invited him as a guest speaker in 1932 (Shewhart, 1933), and acted as promoter of statistical research ${ }^{7}$. The debate led the players to compare their findings with data produced in fields other than the telephone, notably agriculture, the textile industry, metallurgy and building materials. The questions discussed extended beyond the subject of the process control - how to represent quality, how to retain the value of evidence of data series (A.S.T.M., 1933), how to deal with the preoccupation of efficiency, or how to put the concepts into practice.

In the world of the firm, the economic dimension of knowledge operations cannot be ignored. Here we saw it explicitly represented in the acceptance sampling procedures, where the cost of inspection is the major problem. We saw it also as a regulating scheme of the producer-client relation established on a regular basis, where the inspection costs are supported by the producer - while the client accepts a fixed risk of receiving a lot of insufficient quality. It also appears that the most economic inspection scheme, although not suitable for shopfloor practice, is the sequential sampling, an extreme form of a dialogue between the inspector and the lot to be inspected. The empirical machinery is comprised of numerical tables where the most economical procedures are pre-computed, leaving the inspector with nothing to decide except some basic parameters. The procedures appear as "blackboxes" firmly grounded in theory and heavy calculations. In the case of statistical process control, it can be observed a strange mix of refined scientific reasoning and experimentation along with empirically grounded decisions concerning important standard numerical values. The choice of such values as "three sigma" is at the end justified by the general opinion that "it works", but also by the fact that it becomes a convention necessary to the industrial exchange of goods ${ }^{8}$.

\footnotetext{
${ }^{6}$ In the case of a normal distribution, the probability of a false alarm is .003.

${ }^{7}$ After Shewhart's visit to London, the B.S.I. sponsored the work of eminent statisticians and published a handbook on Quality control -viewed from the British side.

${ }^{8}$ A convention named "six sigma" is nowadays implemented in microelectronics industry.
} 


\section{CONCLUSION}

Our goal was to study a knowledge creation process inside a firm in order to better understand the conditions of its success. We chose the case of Statistical Quality Control, which is generally recognized as a success - that is, it brought a new kind of knowledge which is useful to, and even unavoidable for many industries. We hypothesized that its success derives from some capacities possessed by this corpus of methods and tools to respond to requirements important for a firm and its management. On account of prior studies of the topic (Bayart, 1996 ; 2000), we selected three aspects of management requirements which the SQC tools and methods have taken into account: the time factor, the cost factor, the workforce capabilities.

But we also argued that there was something peculiar in the knowledge produced by SQC that should impact its integration in the organization of a firm : "facts" are not easily perceptible, they need equipment and procedures to be established, they present difficult decisions about what level of quality is acceptable. The probabilistic reasonings also are often uneasy to develop and to put to practice. We were, in a general manner, interested in finding the consequences for the firm and its knowledge creation processes of the "probabilistic revolution" which has been celebrated among the historians of sciences and philosophy (Hacking, 1990 ; Kruger \& al, 1988 ; Porter, 1986). Following sociologists of the sciences (Knorr Cetina, 1999), we assumed as a working hypothesis that the peculiar properties of probabilistic knowledge were embodied in the cognitive tools and working procedures practiced inside the firm, i.e. the "empirical machinery" that sustains and structures the relation with empirical facts.

Thus, the plan for our study was to analyze the empirical machinery in order to understand how the two kinds of conditions - the epistemic properties of knowledge and the managerial context - are brought together. We brought out five properties of the SQC knowledge, the first two being a general character of knowledge but strongly marked in the case of SQC : (1) facts are theoretically constructed, (2) (scientific) representations of facts are highly important for the activity, (3) at times the knowledge produced is negative, i.e. it aims at eliminating the phenomena observed, (4) the state of statistical control is a condition for SQC knowledge to be valid but it is fragile, (5) the approach to the state of statistical control, which is also 
the exploration of the assignable causes of variation, is sequential, and can be described metaphorically as a dialogue with the facts.

The industrial context has been considered by the SQC movement in at least three major aspects which have been studied here: (1) a short turnaround time for useful control information in order to allow action on the system, (2) how to optimize the balance between the costs and advantages of control, (3) how to design easy-to-use cognitive tools for a workforce not trained in statistics. In the empirical machinery, only the main elements have been studied here, comprising the Shewhart control chart and the numerical tables of sampling inspection by Dodge \& Romig.

The sampling inspection tables show a good example of a cognitive tool which brings together economic optimality (in terms of cost of inspection), agreement by the inspection workforce (especially for the double sampling plan, opposed to the multiple or sequential sampling plan), technical performance of the sampling plan (a consumer's risk scientifically guaranteed), economic incentive on the provider to maintain a contractual level of quality. But as a counterpart, this tool is a blackbox totally pre-computed (Norman, 1993). Its use, from a theoretical point of view, is that of a procedure of decision, not that of a knowledge production tool: "In lot-by-lot acceptance sampling, the immediate practical question is what to do with the lot. A decision must be made to accept a lot or to reject it or perhaps to screen it. Definite criteria are established to settle such decisions. Usually, the estimation of lot quality from sample quality is viewed merely as an academic question" (Grant \& Leavenworth, 1972, 385). We conclude that sampling inspection methods are not, all by themselves, knowledge creation tools but rather operational procedures grounded in immutable decision rules.

In contrast, the Shewhart control chart is an "open" device, designed to raise questions. The approach to bring the production process to a state of statistical control is a knowledge creating process in the sense that it leads to explore the causes of variability acting upon the production process. The goal however is not the knowledge of these causes per se, but mainly to remove them -and this changes the scope of the knowledge, if not its content. The (statistical) knowledge directly bearing on the production process is represented by the control chart and its control limits: that is all the "theory of the system" that is needed, at least from a statistical viewpoint. 
The process of eliminating the assignable causes of variation is achieved through a sequence of interactions between the theory of the system and its empirical properties. This is what we called the exploratory dialogue, of which the basic elements are the "Shewhart cycles". Like sampling inspection plans, the control chart is pre-computed, but only partly: the control limits result from the "three sigma" rule which has become a general convention. This pre-computed part appears to draw a line between two very different uses of the chart, the one just referred to and the other, which we named the normative dialogue, being to check that the production process is in control. There, the control limits are considered as given norms, not as a means of improving the regularity of the production process. Hence the normative dialogue is not a knowledge creating process but a procedural form of control.

We also met with a different kind of dialogue which we called institutional, meaning that the facts/theory dialogue is mediated by institutional factors such as measurement conventions or labelling standards. The main function of such a mediated dialogue is to construct and ensure intercomparability of the reported facts, from one firm to another firm or industrial sector. This way, local knowledge produced within a single firm can be linked to other knowledge produced by other firms, notably partners in business exchange. Quality conventions play an essential part in defining the nature of goods subject to exchange.

Our case study suggests two conclusions for research on knowledge management.

First, it is critical to consider the nature of knowledge, its "fundamentals" (Weick, 2001), when studying knowledge creation. Each domain of knowledge has its own special properties which make it different from the others. This should be recognized in order to manage knowledge more efficiently. The case of SQC shows that knowledge and methods of a probabilistic nature are often converted into deterministic procedures, derived from the calculation of optimal strategies in front of random events. This is legitimate in some cases but not in all, especially when intelligence has to be brought in to investigate the assignable causes of variation. We also need ethnographic observations of knowledge creation practices to characterize adequately the epistemic culture of the various domains of knowledge inside firms. A bias stems from the material used, which reflects the views of statisticians or organizers, i.e. people in charge of the design. Studying knowledge as practice-based (Brown and Duguid, 1991) is crucial to assess the efficiency of any empirical machinery, the uses of which must be observed in situation. 
Secondly, to return to the "relevance debate" (Starkey \& Madan, 2001), note there are important differences between methods for producing knowledge in academic context and in the context of the firm. In the context of the firm, scientific truth is not the only criterium of importance to management: we saw the pregnancy of delay, cost and work organization considerations. These factors have been explicitly integrated in theorizing, giving rise to original methods not previously known in the academic setting. Academic researchers often find themselves in situations where research means (time, funds, personnel...) are constrained more than expected, but this is usually considered as a matter of fact, not deserving specific theoretical elaboration. This illustrates some difficulties of knowledge transfers from academia to business. In our example, SQC knowledge is produced locally, inside the firm, as it pertains to the production processes and is connected to specific situations. Academia might contribute methodological resources, but certainly not the production of directly useful knowledge. The Bell Labs possessed all the necessary abilities to develop fully the statistical methodology, thus grounding the whole knowledge production process inside the firm. A more ordinary firm has to import many of the necessary knowledge production tools/methods and put them to use, i.e. to locally produce useful knowledge. This raises questions about the possible fit between these tools/methods and the internal organization of the firm and its managerial orientations. Are knowledge-production tools/methods able to circulate among firms and be effective at any place ? Or should we distinguish different classes of knowledge, analyzing their fundamentals and specifying their possibilities of transfer? Viewing knowledge production processes as fact-theory dialogues organized inside the firm with its own resources and in a close fit with ordinary working situations may provide new insights to further academic research.

\section{ACKNOWLEDGEMENTS}

I acknowledge the patient and valuable support received from my colleagues at Centre de recherche en gestion, notably from Anni Borzeix, Sylvain Bureau, Hervé Dumez for their editorial help and suggestions. I am also deeply grateful to Paul Duguid, whose clever advice immensely improved the readability of this paper. Thanks also to the present journal for providing three very competent anonymous 
reviewers whose remarks where a great help and encouragement. Naturally, all remaining weaknesses are my own responsibility.

\section{REFERENCES}

A.S.T.M., American Society for Testing Materials, 1933, Manual on Presentation of Data. 2nd printing, 1937.

Bayart, Denis, 1996, "Savoir organisationnel, savoir théorique et situation: le contrôle statistique sur échantillons," Entreprises et Histoire, (13): 67-82.

Bayart, Denis, 2000, How to make chance manageable: Statistical thinking and cognitive devices in manufacturing control. In Myriam R. Levin (ed.), Cultures of Control. Harwood Academic Publishers, pp. 153-176.

Boltanski, Luc, and Laurent Thévenot, 1991, De la justification. Les économies de la grandeur. Paris: Gallimard.

Brown, John Seely, and Paul Duguid, 1991, "Organizational learning and communities-of-practice: Toward a unified view of working, learning, and innovation," Organization Science, 2: 40-57.

Deming, W. Edwards, 1986, Out of the crisis: Quality, productivity and competitive position. Cambridge, MA: Cambridge University Press.

Dodge, Harold F., 1969, "Notes on the evolution of acceptance sampling plans, parts I-IV," Journal of Quality Technology, 1 (1969), 77-88, 155-162, 225-232; 2 (1970), 1-8.

Fagen, M.D., 1975, A history of engineering and science in the Bell System: The early years (1875-1925). New York: Bell Telephone Laboratories.

Goffman, Erving, 1974, Frame analysis: An essay on the organization of experience. New York: Harper \& Row.

Grant, Eugene L., and Richard S. Leavenworth, 1972, Statistical quality control. International Student Edition, 4th ed: McGraw Hill.

Hacking, Ian, 1990, The taming of chance. Cambridge: Cambridge University Press.

Juran, Joseph M, 1997, "Early SQC: Historical supplement." Quality Progress, Sept: 73-81. 
Klein, Judy L., 2000. Economics for a client: The case of statistical quality control and sequential analysis. In R. E. Backhouse, and J. Biddle (eds.), Towards a history of applied economics. Durham and London: Duke University Press, pp. 27-69

Knorr Cetina, Karin, 1999, Epistemic cultures. How the sciences make knowledge. Cambridge: Harvard University Press.

Kruger, Lorenz, et al, 1988, The probabilistic revolution. Cambridge, London: MIT Press.

Lovitt, Michael R., 1997, "The new pragmatism: Going beyond Shewhart and Deming." Quality Progress, 30: 99-105.

Neyman, J., and E.S. Pearson, 1928, "On the use and interpretation of certain test criteria for purposes of statistical inference, parts 1 \& 2." Biometrika, XX: 175-240, 263-294.

Nonaka, Ikujiro, and Hirotaka Takeuchi, 1995, The knowledge-creating company: How Japanese companies create the dynamics of innovation. New York: Oxford University Press.

Norman, Donald A., 1993. Les artefacts cognitifs. In B. Conein, and N. Dodier c(eds.), Les objets dans l'action. Paris: Ed. de l'EHESS, pp. 15-34.

Porter, Theodore, 1986, The rise of statistical thinking, 1820-1900. Princeton: Princeton University Press.

Sacks, Harvey, E.A. Schegloff, and G. Jefferson, 1974, "A simplest systematics for the organization of turn-taking in conversation." Language, 50: 696 - 735.

Shewhart, Walter A., 1924, "Some applications of statistical methods to the analysis of physical and engineering data." Bell System Technical Journal, III: 43-87.

Shewhart, Walter A., 1931, Economic control of quality of manufactured products. New York, London: Van Nostrand, Mac Millan.

Shewhart, Walter A., 1933, "The role of statistical method in economic standardization." Econometrica: 23-35.

Shewhart, Walter A., 1939, Statistical method from the viewpoint of quality control. Washington: The Graduate School, U.S. Department of Agriculture.

Starkey, Ken, and Paula Madan, 2001, "Bridging the relevance gap: Aligning stakeholders in the future of management research." British Journal of Management, 12(Supplement 1): S3-S26

Stigler, Stephen M., 1977, "Eight centuries of sampling inspection: The trial of the Pyx." Journal of the American Statistical Association, 72: 493-500. 
Taylor, Frederick W., 1911, The Principles of scientific management. New York: Harper Bros.

Tufte, Edward R., 2001, The visual display of quantitative information. 2nd ed. Cheshire, Conn.: Graphics Press.

Tukey, John, 1977, Exploratory data analysis. Reading, MA: Addison-Wesley Publishing Company.

Weick, Karl E., 2001, "Gapping the relevance bridge: Fashions meet fundamentals in management research." British Journal of Management, 12(Supplement 1): S71-S77. 